\title{
Lobectomy versus stereotactic body radiotherapy for stage I non-small cell lung cancer: Post hoc analysis dressed up as level- 1 evidence?
}

\author{
Bryan F. Meyers, MD, MPH, Varun Puri, MD, MBBS, MSCI, Stephen R. Broderick, MD, MPHS, \\ Pamela Samson, MD, Kathleen Keogan, BS, and Traves D. Crabtree, MD
}

\footnotetext{
From the Division of Cardiothoracic Surgery, Washington University in St Louis, St Louis, Mo.

Disclosures: Dr Meyers served on the Data Safety and Monitoring Board of the STARS trial that is the subject of the submitted editorial. As a member of the DSMB, Dr Meyers was compensated by Accuray, the sponsor of the trial. Dr Meyers also reports consulting and lecture fees from Varian.

Received for publication June 18, 2015; accepted for publication June 23, 2015; available ahead of print Aug 7, 2015.

Address for reprints: Bryan F. Meyers, MD, MPH, Washington University in St Louis, Queeny Tower Ste 3108, One Barnes Jewish Hospital, St Louis, MO 63110-1013 (E-mail: meyersb @wustl.edu).

J Thorac Cardiovasc Surg 2015;150:468-71 $0022-5223 / \$ 36.00$

Copyright (C) 2015 by The American Association for Thoracic Surgery http://dx.doi.org/10.1016/j.jtcvs.2015.06.086
}

Many of us in the lung cancer community noted with great interest the publication of the combined outcomes of patients enrolled in 2 failed clinical trials that had each pitted pulmonary resection against stereotactic radiation therapy for operable stage I non-small cell lung cancer. ${ }^{1}$ The article was accompanied by a congratulatory editorial commen$\operatorname{tary}^{2}$ that uncritically echoed the article in broad strokes and called for more effort to conduct and complete carefully planned and monitored clinical trials. Although the authors agree about the importance of evidence, the combined effect of the article by Chang and colleagues ${ }^{1}$ and the accompanying editorial $^{2}$ is a slippery slope of quasi-evidence that adds little to the preexisting dataset except some attractive sound bites that can be delivered out of context and lead to erroneous conclusions.

Management of stage I lung cancer in high-risk patients is controversial. There is a great and growing body of work analyzing and discussing this controversy. Unfortunately, the main focus of this discussion has been the analysis of care provided in routine clinical practice, and the notion of selection bias continues to plague any attempt to decode datasets documenting such routinely delivered clinical care. We and others have used many statistical tools, including multivariable regression models and propensity matching, in attempts to minimize or neutralize such selection bias, but all agree that a large, completed randomized controlled trial (RCT) analyzed according to intention to treat would be the best solution.

The acknowledged need for level-1 evidence in the form of an RCT is signified by the development of 3 such trials several years ago. The American College of Surgeons Oncology Group (ACOSOG), a National Cancer Institute-funded cooperative group now absorbed by

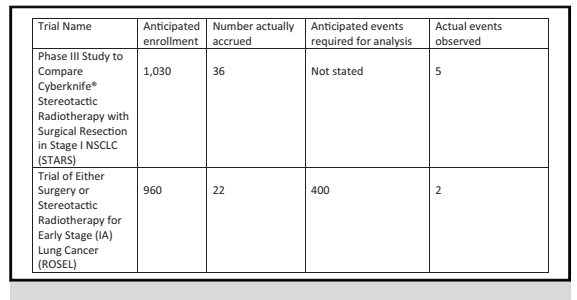

STARS accrued $3.5 \%$ of expected, and ROSEL accrued $2.3 \%$. A pooled analysis remains underpowered.

\section{Central Message}

A pooled analysis of the failed STARS and ROSEL trials is underpowered, producing a randomized but unstable and invalid comparison between SBRT and surgery.

See Editorial Commentary page 472.

the Alliance for Clinical Trials in Oncology, teamed with the Radiation Therapy Oncology Group (RTOG), another National Cancer Institute-funded cancer research cooperative, and together they devised "ACOSOG Z4099/RTOG 1021: A Randomized Phase III Study of Sub-Lobar Resection Versus Stereotactic Body Radiation Therapy in High-Risk Operable Patients With Stage I NSCLC." 3 That study targeted 420 patients with 210 in each treatment group in a noninferiority design to detect a $>10 \%$ difference in 3-year survival. Despite being open by more than 35 centers, the study was closed in May 2013 after 24 months of poor accrual with no analyzable outcome data.

Two other trials (that were not limited to high-risk patients) met the same fate, the Phase III Study to Compare Cyberknife Stereotactic Radiotherapy with Surgical Resection in Stage I NSCLC (STARS) and the Trial of Either Surgery or Stereotactic Radiotherapy for Early Stage (IA) Lung Cancer (ROSEL). The small patient dataset that was generated from these 2 trials forms the basis of the article by Chang and colleagues. ${ }^{1}$ It is natural to want to draw on data generated from a randomized trial. Such data is expensive to obtain, and diligent efforts to analyze and share such data is appropriate and respectful to the trial participants who sacrificed some degree of personal choice to allow themselves to be randomized. 
However, the notion of randomization is only 1 element describing quality of data and the simple fact that data were collected in 2 failed randomized trials may not overcome the many limitations posed by this pooled dataset and its subsequent analysis. This editorial attempts to warn against overstating the value of the salvaged data by pointing out some limitations. The goal is to make sure readers understand the persisting complexity of the choice between surgery and stereotactic body radiation therapy (SBRT) and how little this recent article $^{1}$ has done to clarify the issue or change the debate.

Statistical analysis in that article ${ }^{1}$ neglected to tell the reader about the sample size that was believed to be necessary at the outset of the 2 trials. In the Netherlands, ROSEL required a sample size of 960 patients to test whether radiosurgery "was not inferior to surgery." ${ }^{4}$ The framers of the study estimated that 200 events (ie, deaths) per arm would be needed to complete the primary aim. Instead of the required 960 enrolled patients and 200 events per arm estimated to be needed to analyze the trail, ROSEL recruited 22 patients and recorded exactly 1 event in each arm of the study within 3 years.

The STARS trial had an estimated sample size of 1030 to detect a hazard ratio of death of $>1.5$ when comparing the inferior therapy to the superior therapy. ${ }^{5}$ When the study was closed for poor accrual, 36 of 1030 patients had been enrolled and randomly assigned therapy. The fact that neither study achieved $4 \%$ of the expected sample size is notable, yet this fact was completely left out of the article by Chang and colleagues ${ }^{1}$ and the editorial. ${ }^{2}$ What are the implications of the small sample size? Clearly, instability of the results is one major risk. Of the 4 distinct groups that are naturally evident (STARS surgery, STARS radiotherapy, ROSEL surgery, and ROSEL radiotherapy), members of 3 of the 4 groups experienced 3-year mortality estimates that are lower than anticipated. There was a single death in each of the 2 arms of ROSEL and none at all in the STARS radiotherapy group. However, the STARS surgery group had a higherthan-expected hazard of death, and that worse-thanexpected outcome in the 16-patient surgical cohort drives the entire analysis.

This collection of patients, although generated from random assignments of therapy, differs in important ways from an RCT. The analysis and monitoring of a clinical trial is conducted in such a way to protect the patients, yet, at the same time, reduce the chances of a falsely positive result. Trialists do not want to continue randomizing subjects if one arm is clearly superior, but they are wary about proclaiming a difference too quickly. One way that this is done is through group sequential analysis and predesignated planned interim analyses of the data. In the very early stages of these planned analyses, the threshold to declare a positive result is very conservative to avoid making too much out of early differences seen in small sample sizes after short follow-up. One example of these strategies to avoid misinterpretation of early differences in small sample sizes is the O'Brien-Fleming group sequential boundaries. The use of such techniques "has the desirable property of being very conservative early, when one would be skeptical of unstable efficacy and safety results and where data are inadequate to address key model assumptions." ${ }^{6}$ An article such as that by Chang and colleagues, ${ }^{1}$ created with unlimited looks at the outcome data from closed and failed trails, has a much higher risk of a type 1 error and may interpret statistical variability as a signal rather than noise. It is likely that the entire moving force to publish this work is the large and unexpected difference seen in the small cohorts of STARS surgery and STARS SBRT groups. Without that surprise difference, detected after study closure and without the constraint of a data and safety monitoring process applicable to ongoing trails, there is no story here.

An important implication of small size is the potential overweighting of rare events in small cohorts of patients. The mortality rate in the surgical arm of this post hoc collection of normal-risk patients is 1 out of 27 or roughly $4 \%,{ }^{1}$ which is 2 to 4 times the expected mortality of normalrisk surgical resection patients for stage I lung cancer. Fernando and colleagues ${ }^{7}$ reported on the outcomes of a prospective trial in patients with stage I non-small cell lung cancer deemed high risk by objective criteria. The surgical mortality in that cohort was 3 out of $222(1.4 \%)$. Our own group, led by Puri, ${ }^{8}$ applied the ACOSOG objective high-risk criteria to more than 1000 patients treated for stage I non-small cell lung cancer and observed 1\% mortality in the so-called high-risk patients and $2 \%$ in those who did not meet the high-risk criteria. Kozower and colleagues analyzed the Society of Thoracic Surgeons National Cardiac Surgery Database to report on lobectomies performed at 56 sites, with a mortality of 71 out of $4979(1.4 \%) .{ }^{9}$ The fact that there was only 1 treatment-related death in the combined surgical cohort of the 2 failed trials reported by Chang and colleagues ${ }^{1}$ seems hard to quarrel with, but 1 death in 27 patents, when the expected treatment mortality rate is between 1 in 50 or 1 in 100, is testimony for the need for a larger study.

A substantial risk of the small size is the threat to generalizability. Because both of the merged, failed trails closed after $<4 \%$ completion due to inability to accrue patients, one must wonder about the degree to which these enrolled patients are representative of the overall universe of patients the authors aim to represent. If we sent out a survey and got back $4 \%$ of the mailed surveys, there would be justifiable concern about the degree to which the respondents represent the total group queried. Because the failure to accrue suggests that either the patients or the treating 
doctors did not have equipoise in most cases, the extent to which these 58 patients resemble, or differ from, the majority of patients who refused randomization would inform us of the wisdom of extending what little findings there are here to future patients. Most likely, those refusing randomization were treated off trial with either surgery or SBRT at the same institutions and by the same practitioners, yet some unstated factors made most patients unwilling to be randomized.

Another problem with a small study is the fact that there are numerous adverse outcomes that might occur, and the small sample size impairs the ability to see patterns that make the overall result seem plausible. If 1 therapy is superior, that superiority would be gained from 1 of the following factors: lower early treatmentrelated mortality, better local control, better regional control, fewer distant metastasis, and lower late influence of therapy on comorbidities and noncancer deaths. In this collection of 58 patients, ${ }^{1}$ there was 1 treatment-related death in the surgical group $(4 \%)$ and none in the SBRT group. After that, Chang and colleagues' Figure $1^{1}$ shows no differences whatsoever between the 2 groups with regard to local recurrence, regional recurrence, or distant recurrence. How can these facts be consistent: only 1 treatment death, no discernable differences in any of the modes of recurrence, yet a claim made in the article $^{1}$ and echoed in the accompanying editorial ${ }^{2}$ of a statistically significant difference of survival favoring the SBRT group? The answer must be the small sample size and the instability of the results when frequencies of adverse events are low.

Based on the data provided, there is no clear ability to discern the degree of surgical risk for these patients or the surgical efficacy of the treating sites. The fact that they are described as "operable" is a broad and subjective claim and is not useful. An 88-year-old with emphysema and a 3-cm lower lobe tumor abutting the visceral pleura and the esophagus is operable, just as a 55-year-old former smoker with normal lung function and a $9-\mathrm{mm}$ spiculated lesion in the right middle lobe is operable. However, those 2 patients have far different life expectancies and unique issues that inform their treatment selection. Granted, "operable" is a hard description to make, and we have struggled to add objectivity and clarity to that term in other work. For cardiac surgery patients, there is the European System for Cardiac Operative Risk Evaluation and the Society of Thoracic Surgeons risk predictor. It is likely that future trials would be wise to have a granular and reproducible method of assessing and documenting risk that goes beyond subjective surgeon determination of operability. The thoracic surgery community has the data now to calculate an expected perioperative mortality risk, and such data should be used as inclusion criteria and be reported in efforts to demonstrate the pretreatment comparability of treatment cohorts.

In the article by Chang and colleagues, ${ }^{1}$ there are no data or discussion about the effectiveness of the care provided to the surgical group. STARS enrolled 36 patients at 28 sites, whereas ROSEL enrolled 22 patients at 10 sites. It seems that most participating sites would have enrolled 1 or 2 patients, so it is impossible to know much about the quality of work done. There is collective belief and trial evidence to suggest that treatment of patients with stage II or stage III lung cancer with adjuvant chemotherapy improves their survival. There is also evidence to show that clinical staging underestimates a substantial fraction of patients with clinical stage I disease by missing occult hilar and mediastinal lymph node metastases. Stiles and colleagues ${ }^{10}$ studied 266 patients who underwent resection with clinical stage Ia tumors and found $11.7 \%$ to be node positive despite good clinical staging. The risk for occult nodal mets is increased with tumor size, and the likelihood of finding such metastatic disease is proportional to the effort spent searching for it by removing nodes. Chang and colleagues ${ }^{1}$ do not tell us how many patients had "good surgery" as defined by systematic sampling or node dissection, and how many were appropriately treated with chemotherapy for occult metastases that were unmasked. The fact that $80 \%$ of the resections in this combined series were via thoracotomy is also in contrast to the fact that more than half of stage I lobectomies in the United States are done via thoracoscopy.

Finally, there is lack of clarity in the reporting of the main results: Figure 2, A (overall survival) and the accompanying text in the article by Chang and colleagues ${ }^{1}$ both give the hazard ratio for overall survival as 0.14 favoring radiation therapy with a $95 \%$ confidence interval ranging of 0.017 to 1.190 , and a $\log$-rank $P$ value of .037. By reporting a hazard ratio, the authors imply that a Cox regression was used. The log-rank test must refer to a comparison of the Kaplan-Meier survival curves. It is not clear how the $P$ value for the log-rank test of inference is less than .05 for the differences between the survival curves, but the $95 \%$ confidence intervals for the hazard ratio include unity. A $P$ value for the reported hazard ratio would have to be greater than .05 , but it was omitted. This inconsistency exists even if we accept a probability of a type I error as $5 \%$, but this issue becomes more concerning for falsely rejecting the null hypothesis when we consider inflated alpha of the unplanned and frequent access the authors had to the data. Regardless of those details, it is very risky to make any survival claims when the studied population is $<4 \%$ of the size deemed necessary by skilled statisticians at the outset, and when 25 of the 58 enrolled patients had 
neither had an event nor reached the 3-year mark in their follow-up.

So, what is the value of an early analysis of a post-hoc combination of 2 failed trials? The accompanying editorial tried to point to some value here:

Nevertheless, the results suggest that SABR is not inferior to surgery, with a hint that it might be better in terms of clinical effectiveness. The findings cast doubt over the superiority of lobectomy, sufficient to suggest group equipoise (a prerequisite for future randomised trials), and the investigators state that further trials in this setting are warranted. ${ }^{2}$

It is ironic that the congratulatory editorial ${ }^{2}$ states that this article ${ }^{1}$ suggests group equipoise. Was there not already group equipoise when the ROSEL trial and the STARS trial randomized those 58 patients and screened many hundreds more? Similarly, sufficient equipoise allowed ACOSOG 4099/RTOG 1021 to randomize patients at elevated risk for death and complications between sublobar resection and SBRT, but that trial faced a similar failure due to the same lack of accrual that led to the closure of ROSEL and STARS. There seems to be no shortage of equipoise when creating these trials, because there are at least 2 more that are in the initial stages of organization. The Sublobar Resection Versus Stereotactic Ablative Radiotherapy for Lung Cancer trial will randomize between sublobar resection and stereotactic ablative radiotherapy. ${ }^{11}$ Another trial within the Veterans Administration system is hinted at, but there is not a current entry on ClinicalTrials.gov nor any substantial information available online. With 3 failed trials and possibly 2 additional trials in progress, perhaps this small and imperfect article by Chang and colleagues ${ }^{1}$ will allow some of the equipose trickle down to the patients and their families who have been unwilling to consent to randomization.

An unplanned publication initiated after extensive review of the outcomes and discovery of an interesting survival trend has a weight that is far below that of a successfully completed phase III trial. Although these patients have been randomized, Chang and colleagues ${ }^{1}$ do not present a prospective and randomized trial. "Operable" stage I lung cancer is vague and to use that to describe a group of patients to whom this recent article applies is to paint with too broad of a brush. There is plenty of existing equipoise about the decision of surgery or SBRT in patients who face increased risk for complications and death after surgery. Readers should be very careful about providing soundbites or snippets of results from the study without a full consideration of the deficiencies of the article.

\section{References}

1. Chang JY, Senan S, Paul MA, Mehran RJ, Louie AV, Blater P, et al. Stereotactic ablative radiotherapy versus lobectomy for operable stage I non-small-cell lung cancer: a pooled analysis of two randomized trials. Lancet Oncol. 2015;16: 630-7.

2. Treasure T, Rintoul RC, Macbeth F. SABR in early operable lung cancer: time for evidence. Lancet Oncol. 2015;16:597-8.

3. Fernando HC, Timmerman R. American College of Surgeons Oncology Group Z4099/Radiation Therapy Oncology Group 1021: A randomized study of sublobar resection compared with stereotactic body radiotherapy for high-risk stage I non-small cell lung cancer. J Thorac Cardiovasc Surg. 2012;144:S35-8.

4. Randomized Study to Compare CyperKnife to Surgical Resection in Stage I Non-Small Cell Lung Cancer (STARS). Available at: https://clinicaltrials.gov/ archive/NCT00840749/2009_02_09. Accessed June 2015.

5. Trial of Either Surgery or Stereotactic Radiotherapy for Early Stage (IA) Lung Cancer (ROSEL). Available at: https://clinicaltrials.gov/ct2/show/NCT00687986. Accessed June 2015 .

6. Ellenberg SS, Fleming TR, DeMets DL. Statistical, Philosophical and Ethical Issues In Data Monitoring Committees in Clinical Trials: A Practical Perspective. Hoboken, NJ: John Wiley \& Sons Ltd; 2002.

7. Fernando C, Landreneau RJ, Mandrekar SJ, Hillman SL, Nichols FC, Meyers B, et al. Thirty and ninety day outcomes after sublobar resection with and without brachytherapy for non-small cell lung cancer: results from a multicenter phase III study. J Thorac Cardiovasc Surg. 2011;142:1143-51.

8. Puri V, Crabtree TD, Bell JM, Kreisel D, Krupnick AS, Broderick S, et al. National Cooperative Group trials of "high-risk" patients with lung cancer: are they truly "high-risk"? Ann Thorac Surg. 2014;97:1678-85.

9. Kozower BD, Sheng S, O'Brien SM, Liptay MJ, Lau CL, Jones DR, et al. STS database risk models: predictors of mortality and major morbidity for lung cancer resection. Ann Thorac Surg. 2010;90:875-81.

10. Stiles BK, Servais EL, Lee PC, Port JL, Paul S, Altorki NK. Clinical stage IA non-small cell lung cancer determined by computed tomography and positron emission tomography is frequently not pathologic IA non-small cell lung cancer: the problem of understaging. J Thorac Cardiovasc Surg. 2009;137:13-9.

11. JoLT-Ca Sublobar Resection (SR) Versus Stereotactic Ablative Radiotherapy (SAbR) for Lung Cancer (STABLE-MATES). Available at: https://www clinicaltrials.gov/ct2/show/NCT02468024. Accessed June 15, 2015. 